\title{
Valoración de las experiencias educativas en tiempos de COVID-19 una oportunidad para innovar; caso estudiantes de la Universidad Técnica Nacional Sede Guanacaste
}

\author{
Evaluation of educational experiences in times of \\ COVID-19 an opportunity to innovate; case of students of \\ the Universidad Técnica Nacional Sede Guanacaste
}

\author{
Jorge Luis Loáiciga Gutiérrez \\ Universidad Técnica Nacional, Alajuela, Costa Rica. \\ jloaicigag@utn.ac.cr \\ https://orcid.org/0000-0002-0421-3906 \\ Carlos Luis Chanto Espinoza \\ Universidad Nacional, Sede Regional Chorotega Liberia, Costa Rica. \\ carlos.chanto.espinoza@una.cr \\ https://orcid.org/0000-0002-3420-7259
}

Referencia/ reference:

Loáiciga, J. y Chanto, C. (2020). Valoración de las experiencias educativas en tiempos de COVID- 19 una oportunidad para innovar; caso estudiantes de la Universidad Técnica Nacional Sede Guanacaste. Yulök Revista de Innovación Académica, 4(2). 104-113. https://doi.org/10.47633/yulk.v4i2.285

Recibido: 4 de noviembre del 2020

Aceptado:1 de diciembre del 2020

\section{Resumen}

El objetivo de este estudio fue el de valorar el impacto del cambio metodológico del proceso de enseñanza-aprendizaje, de modalidad presencial a remota con ayuda de las tecnologías de información y comunicación (TIC) como consecuencia de la Covid-19, en los estudiantes de la Universidad Técnica Nacional (UTN). El trabajo se desarrolló según un enfoque mixto, de carácter descriptivo e interpretativo. La muestra estuvo conformada por 117 estudiantes de la UTN con sede en la provincia de Guanacaste, Costa Rica. Los resultados revelan oportunidades de mejora en un punto convergente como la conectividad y acceso a dispositivos adecuados para contemplar una modalidad remota con uso de herramientas digitales en igualdad de condiciones. Se concluye que la investigación permite caracterizar la situación institucional y son insumos para la toma de decisiones.

Palabras clave: Tecnologías de la Información y la Comunicación, Educación Superior, Innovación Pedagógica, Enseñanza, Covid-19.

\section{Abstract}

The objective of this study was to assess the impact of the methodological change of the teaching- learning process, from face-to-face to remote mode with the help of information and communication technologies (ICT) as a result of Covid-19, on students of the National Technical University (UTN). The work was developed according to a mixed descriptive and interpretative approach. The sample consisted of 117 UTN students based in the province of Guanacaste, Costa Rica. The results reveal opportunities for improvement in a convergent point such as connectivity and access to adequate devices to contemplate a remote modality with the use of digital tools under equal conditions. It is concluded that the research allows characterizing the institutional situation and are inputs for decision making.

Keywords: Information and Communication Technologies, Higher Education, Pedagogical Innovation, Teaching, Covid-19. 


\section{Introducción}

La pandemia de la Covid-19 nos ha mostrado múltiples escenarios de una serie donde su final era predecible: la llamada educación virtual. En una carrera contra el tiempo, alumnos, profesores y las múltiples instituciones de educación superior apalearon a adaptarse a una modalidad de enseñanza-aprendizaje innovadora, pero no desconocida por muchos.

Esta investigación es un esbozo sobre como la educación superior se ha visto obligada a enfrentar este desafío y cuales han sido, las estrategias de la utilización de las TIC en el proceso de enseñanza-aprendizaje. Se identifican algunas herramientas utilizadas por la Universidad, tratando de hacer frente a la nueva modalidad de enseñanza con la utilización de herramientas tecnológicas y que además asegurarse que el educando este adquiriendo los conocimientos de la forma clara, explícita y práctica.

La educación es un elemento esencial y transformador de la sociedad, es uno de los pilares para mejorar el desarrollo sostenible y nuestras vidas, tal y como lo menciona la Cámara de Comercio Costarricense-Norteamericana de Comercio de Costa Rica (Amcham, 2019, párr. 1):

(...) La educación es la base para mejorar nuestra vida y el desarrollo sostenible. Además de mejorar la calidad de vida de las personas, el acceso a la educación inclusiva y equitativa puede ayudar abastecer a la población local con las herramientas necesarias para desarrollar soluciones innovadoras a los problemas más grandes del mundo.

Como lo establece la Organización de las Naciones Unidas, las instituciones de educación superior están comprometidas a brindar las herramientas imprescindibles donde los educandos se enfrentan a un cambiante mundo globalizado colmado de desafíos, y actualmente en tiempos de Covid-19. Tal y como lo plantea Delgado et al. (2020, p. 50):

Actualmente estamos viviendo una época de cambios, en la cual tanto niños como adolescentes son los que utilizan más tiempo enlazados con las nuevas tecnologías como el smartphone o tables además del uso de programas o aplicaciones móviles; las mismas que pueden estar siendo mal utilizadas.

De la misma forma es importante el acompañamiento y apoyo a los educandos que se encuentran en sus casas, con el fin de obtener un conjunto de cualidades y aspectos en cada educando. Para Mendía y García (2015) “el acompañamiento educativo fomenta la educación en valores, autoestima, competencia social y ciudadana y el emprendimiento social, el diálogo, la acción cooperativa y la toma de decisiones" (p. 49).

A su vez indicar el gran esfuerzo de cada académico sometido a capacitaciones para poder hacer uso de las diferentes plataformas educativas virtuales disponibles y hacer llegar el aprendizaje a los estudiantes.

Aguilar (2020, p. 1) menciona lo siguiente:

El confinamiento por el COVID-19 nos ha dado la oportunidad de reflexionar sobre muchas cosas y, también, hemos comprobado todas aquellas cosas que no funcionan. El profesorado de todos los niveles educativos hemos tenido que adaptar las clases y la investigación para poder seguir trabajando con nuestro estudiantado. Un gran esfuerzo porque ha habido que improvisar, ha habido un problema de acceso a muchos recursos que se han tenido que suplir o que eran insuficientes o inexistentes en algunos casos, y en muchos hogares se ha añadido el problema de tener que cuidar de los hijos e hijas o de personas mayores o dependientes.

La pandemia por efectos de la COVID-19, generó que los docentes universitarios tuvieran que adaptar las clases a una modalidad virtual, con la utilización de herramientas tecnológicas, aunado a un gran esfuerzo por seguir trabajando con el estudiantado, y así alcanzar los objetivos del proceso de enseñanza-aprendizaje.

Tal y como lo menciona Zeballos (2020) “(...) las estrategias tecnológicas evolucionan hacia espacios más formativos promoviendo el aprendizaje colaborativo y la reflexión crítica del uso de las tecnologías" (p. 196).

Por lo tanto, la pandemia causada por el COVID-19 brinda otra perspectiva, según lo menciona Hueso (2020): “(...) el coronavirus supone una oportunidad para dejar atrás una educación obsoleta del siglo XX. Se recuerda la necesidad del cambio hacia competencias digitales y el pensamiento computacional" (p. 1).

Por ende, debemos destacar que a pesar de que la educación superior ha tenido sus conflictos para logar adaptarse a la modalidad online, se destaca que no se ha detenido, todo lo contrario, se está adaptando con el apoyo de las TIC, para lograr el cumplimiento con el proceso de enseñanza- aprendizaje. El reto presente es someter el impacto perjudicial que la Covid-19 ostenta en el marco del aprendizaje y la educación, y así fructificar esta ex- 
periencia para iniciar el nuevo camino de mejora en los aprendizajes.

\section{Referencial teórico}

Debido a la pandemia por Covid-19, la educación superior cambió, y Costa Rica no es la excepción. Donde se han presentado transformaciones radicales de fondo y de forma que algunas universidades no habían considerado llevar a cabo a corto plazo, y mucho menos, de la manera tan abrupta como sucedió a inicio del mes de marzo del 2020. Como menciona GEM (2020), “(...) Todos los países están introduciendo o ampliando las modalidades de educación a una distancia existente basadas en diferentes combinaciones de tecnología ante la pandemia las operaciones globales están confluyendo" (párr 4).

Todo esto dentro del marco de las medidas sanitarias para el contorno educativo, en donde cada país se ha enfocado en garantizar la continuación del proceso de enseñanza-aprendizaje con ayuda de herramientas tecnológicas denominadas TIC, evitando en lo potencial la complicación se han establecido elementos como la incorporación de modalidades existentes de educación a distancia, utilización de plataformas en línea (aulas virtuales), así como cualquier otra herramienta TIC que permita el seguimiento del proceso de enseñanza-aprendizaje.

Tal y como lo menciona la (UNESCO, 2020, p. 2)

(...) Desde el inicio de la crisis, los docentes han desempeñado un papel fundamental para garantizar la continuidad del aprendizaje gracias a la educación a distancia, cuando ha sido posible, y para que se tenga en cuenta el bienestar de los alumnos.

La Universidad Técnica Nacional (UTN) ha tomado ese desafío de brindar una educación global y la responsabilidad de ofrecer herramientas tecnológicas a la comunidad universitaria, buscando enfrentarse a una sociedad que se encuentra en continuo y permanente cambio en tiempos de Covid-19, donde la utilización de las TIC ha permitido reducir los obstáculos de distancia (zonas geográficas), de tiempo y espacio en diferentes escenarios, minimizar el riesgo, entre otras.

Tal y como lo menciona (Sandoval, 2020, p. 29)

(...) en el contexto actual de la modalidad virtual, los docentes han tenido que empezar un proceso de alfabetización funcional en el conocimiento y manejo de las herramientas TIC para poder ser im- plementadas como estrategias didácticas en el proceso ser implementadas como estrategias didácticas en el proceso formativo con sus estudiantes.

Ante este escenario, se han impartido múltiples capacitaciones referentes a la utilización y manejo de plataformas virtuales, tratando de subsanar cualquier desconocimiento. Se implementaron cursos para el manejo de herramientas TIC, como apoyo a la docencia que muchos docentes utilizaron ante la necesidad que imperaba de atender a sus estudiantes; y así continuar en esta tarea tan importante como lo es la docencia.

Ejercicio fundamental, tal y como lo establece (Guaman-Chávez, 2020, p. 22)

Las instituciones educativas mediante el surgimiento y expansión de esta nueva plaga COVID-19, ha exigido cambios inmediatos de 180 grados en menos de unas semanas, obligando a los actores de la actividad educativa utilizar herramientas virtuales de aprendizaje aplicado a la continuidad educativa en sus labores académicas.

El desafío no ha sido sencillo, ya que el gran reto obligó a miles de docentes, estudiantes y administrativos a incursionar en el contexto digital de manera abrupta y así alcanzar concretamente los objetivos y programas establecidos al inicio del semestre, un objetivo titánico, pues nos enfrentamos a las brechas digitales que se hicieron latentes en este proceso de enseñanza- aprendizaje, en donde algunos docentes y educandos no contaban con fácil acceso al Internet por su ubicación geográfica; en otros casos, la reciente condición de desempleo de los padres de familia ante el cierre de negocios debido a las medidas sanitarias, por lo que tuvieron la necesidad de buscar trabajo para apoyar a su familia dejando en segundo plano sus estudios; también, se presentaron algunos casos en los que no era posible para los alumnos pagar el servicio de Internet para ingresar en días y horas determinadas, ante este hecho se buscaron formas para atender estas situaciones.

Sin duda alguna, la pandemia vino a mostrarnos la necesidad de generar consciencia respecto a estas desigualdades y desarrollar una actitud impávida que logre trasmutar los contextos sociales desiguales que se han normalizado con el pasar del tiempo, no obstante, nunca debió ser considerada normal, las condiciones menesterosas en las que viven muchos, la desigualdad política, social y económica, y ser apáticos al presenciar solo una porción de nuestra normalidad social. La notable brecha económica y social 
tienen una estrecha relación con la digital, lo cual afecta arduamente a muchos estudiantes de educación superior.

Según lo menciona Barrera Rea y Guapi Mullo (2018) "Las plataformas virtuales se han convertido en una herramienta potente en la tecnología educativa, siendo capaz de desarrollar la independencia de los saberes en muchos casos y el acercamiento virtual entre docentes y dicentes." (p. 1). Por ende, la tecnología que actualmente, conocemos es la mayor herramienta ante esta pandemia provocada por la Covid-19. En el amplio campo de las innovaciones, las herramientas TIC han dejado una huella permanente en nuestras vidas, de tal forma que si en algún momento esta desaparece dejaría un caos permanente en toda la humanidad.

\section{Metodología}

Hubo una época en el mundo donde la única educación que se conocía era aquella que se daba en aspectos presenciales donde las únicas herramientas eran la tiza y el pizarrón. Desde la antigüedad, los seres humanos han estado en constante evolución, realizando innovaciones en cada uno de los procesos de enseñanza-aprendizaje, con base en lo anterior, De la Iglesia Villasol (2019) menciona lo siguiente: "Hacia escenarios de enseñanza-aprendizaje que traspasan los muros de las aulas físicas hacia plataformas virtuales, abre canales asincrónicos de aprendizaje" (p. 1).

El objetivo de la presente investigación consiste en valorizar el impacto del cambio metodológico del proceso de enseñanza-aprendizaje, de modalidad presencial a modalidad virtual como consecuencia de la Covid-19, en los estudiantes de la Universidad Técnica Nacional Sede Guanacaste.

Para dicho trabajo de investigación se utilizó un enfoque mixto, donde el instrumento utilizado fue la encuesta, para establecer el impacto de la pandemia de la Covid-19 en el proceso pedagógico, la utilización de las TIC en el proceso de enseñanza-aprendizaje de los estudiantes de la Universidad Técnica Nacional Sede Guanacaste, se planteó como el objeto de estudio. De la misma forma, se estableció diagnosticar la incorporación y utilización de las herramientas TIC en el proceso del docente, con el fin de dar a conocer posibles necesidades individuales y colectivas planteadas por parte de los educandos.
En la misma línea, el estudio bibliográfico permitió la edificación del marco teórico en donde se definió la estructura en la investigación, para evaluar el estado del arte concerniente con el uso de las TIC en el proceso de enseñanza-aprendizaje.

\section{Resultados y análisis}

Los resultados obtenidos en el apartado previo describen las características y percepciones por el estudiantado de la Universidad Técnica Nacional, Sede Guanacaste en cuanto a la educación virtual por medio de las TIC en tiempos de Covid-19.

En primera instancia, se determinó el perfil sociodemográfico donde el $64,1 \%$ de la población estudiantil que respondió el cuestionario es de sexo femenino y $35,9 \%$ masculino. Además, que en su mayoría son residentes del cantón de Cañas 28,2 \% y Liberia 24,8 \%, Guanacaste.

También, el promedio de edad se encuentra entre el rango de 20-22 años, siendo este más alto entre la carrera de Gestión y Administración Empresarial (GAE) y Gestión Ecoturística (GE) y el más bajo en la carrera de Ingeniería en Tecnologías de la Información (ITI).

De acuerdo con la información suministrada por los estudiantes encuestados el 76,9 \% manifiesta encontrarse en sus primeras ocasiones cursando clases en modalidad remota con el uso de herramientas digitales que proporcionan las TIC.

En el apartado de conectividad se les preguntó desde dónde se han conectado, principalmente, para realizar sus actividades académicas sincrónicas y asincrónicas en esta nueva modalidad. El mayor porcentaje $83,8 \%$ indica que, desde su casa de habitación, el 33,3 \% usan datos de sus celulares pospagos, $13,7 \%$ datos de celulares prepagos, $4,3 \%$ usan zonas públicas para conectarse y en menor porcentajes $0,9 \%$ acceso desde la casa de un familiar o el trabajo y un $0 \%$ en Laboratorio de la Universidad, esto por motivo de cierre de las aulas por la pandemia del Covid-19. En la figura 1 se presenta una visión general de la brecha digital en temas de conectividad. 


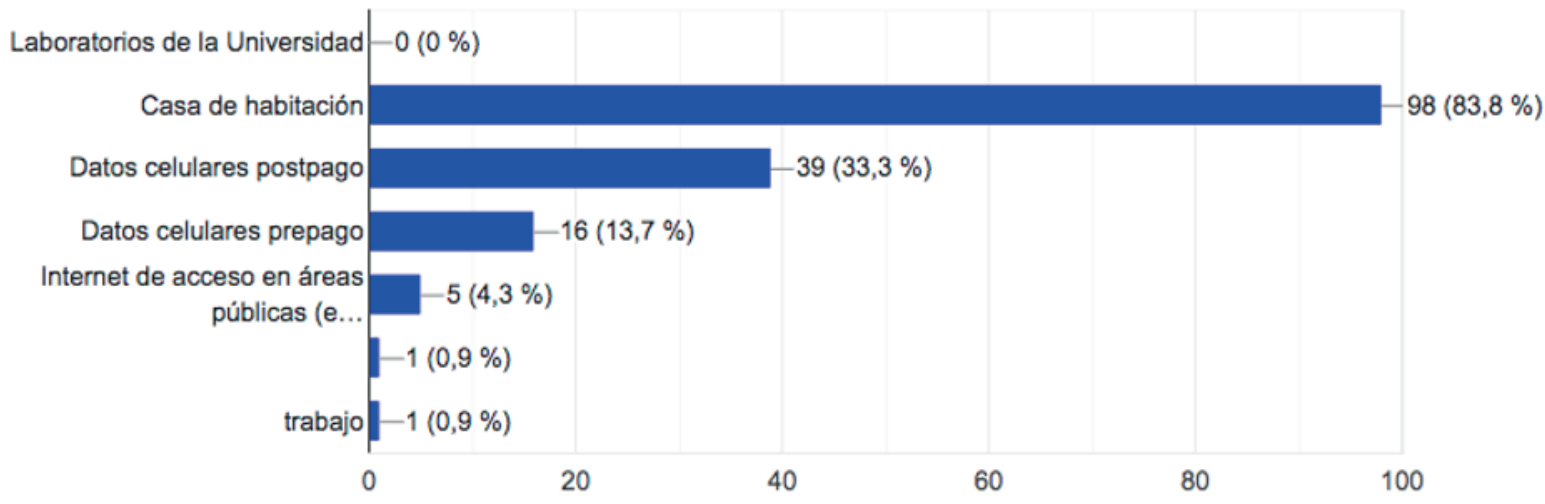

Figura 1. Conectividad en las actividades sincrónicas y asincrónicas. Fuente: elaboración propia.

Otro factor importante es que esta población que indica conectarse desde su casa de habitación el 77,8 \% lo realiza desde una computadora portátil, el $61,5 \%$ desde su teléfono inteligente pospago y el $21,4 \%$ de prepago. Se debe resaltar que solo el 7,7 \% cuenta con una computadora de escritorio y un $0,9 \%$ con una Tablet que conecta al wifi.

Debido a los cambios drásticos en el cambio de modalidad a una remota con herramientas tecnológicas, se le preguntó a la población estudiantil cuánto tiempo en promedio ha dedicado por día a los distintos cursos en actividades asincrónicas y el 39,3\% respondió de 4 a 6 horas, el $31,6 \%$ de 1 a 3 horas y finalmente el $28,2 \%$ más de 6 horas diarias.

Otro aspecto importante por analizar es la plataforma de mediación que mayor han utilizado para recibir las actividades sincrónicas y asincrónicas, esta es Google Meet/ Hangouts con un $97,4 \%$ y el campus virtual de la UTN con un $86,3 \%$. Además, con una importante participación en el uso de WhatsApp con el 74,4 \%, el correo electrónico institucional un $65 \%$, la plataforma de Microsoft Teams representó un $52,1 \%$ y en menor uso las plataformas de Google Classroom con 30,8 \%, Zoom con 29,9 $\%$, One Drive con el 18,8\% y finalmente el $17,9 \%$ para Youtube.

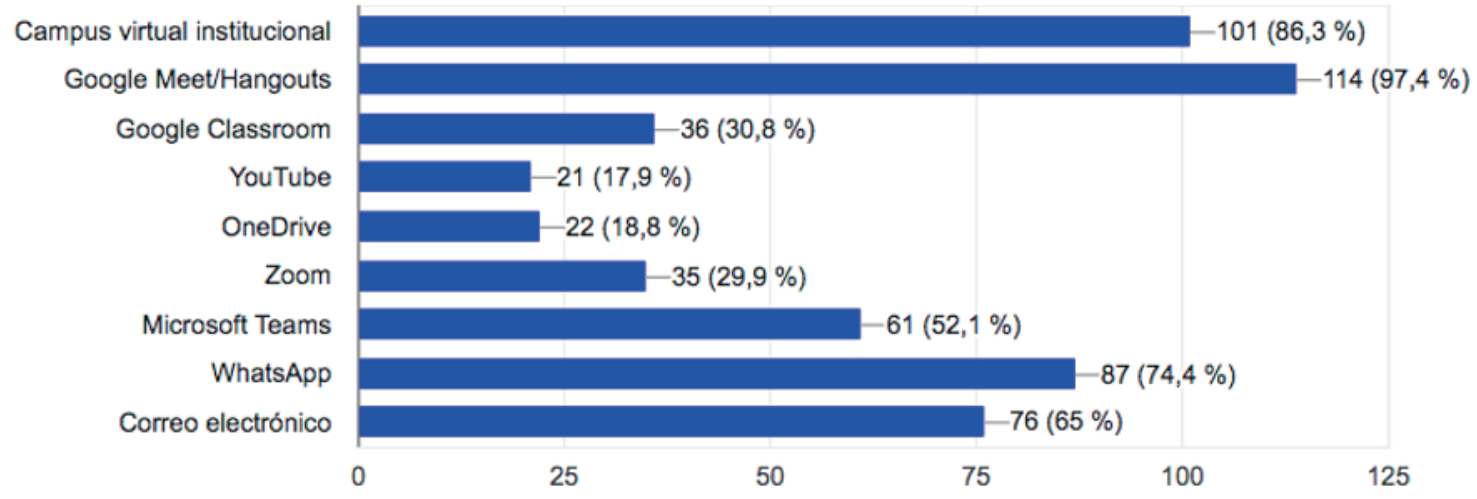

Figura 2. Plataformas de mediación en actividades sincrónicas y asincrónicas. Fuente: elaboración propia. 
Dentro de esta nueva mediación pedagógica se les solicitó a los estudiantes encuestados indicar qué estrategias han implementado los docentes para impartir las clases. Más del $91,5 \%$ indicó la videoconferencia en tiempo real, el $88 \%$ en actividades de tareas en grupo e individuales, un 56,4
$\%$ mediante foros en línea desde el campus virtual de la UTN. El $50 \%$ en videos en la web y lecturas en la web, el $41 \%$ ha utilizado cuestionarios en línea. Por otra parte, solo el $35 \%$ utiliza videos pregrabados y documentos escaneados y el $21 \%$ libros electrónicos.

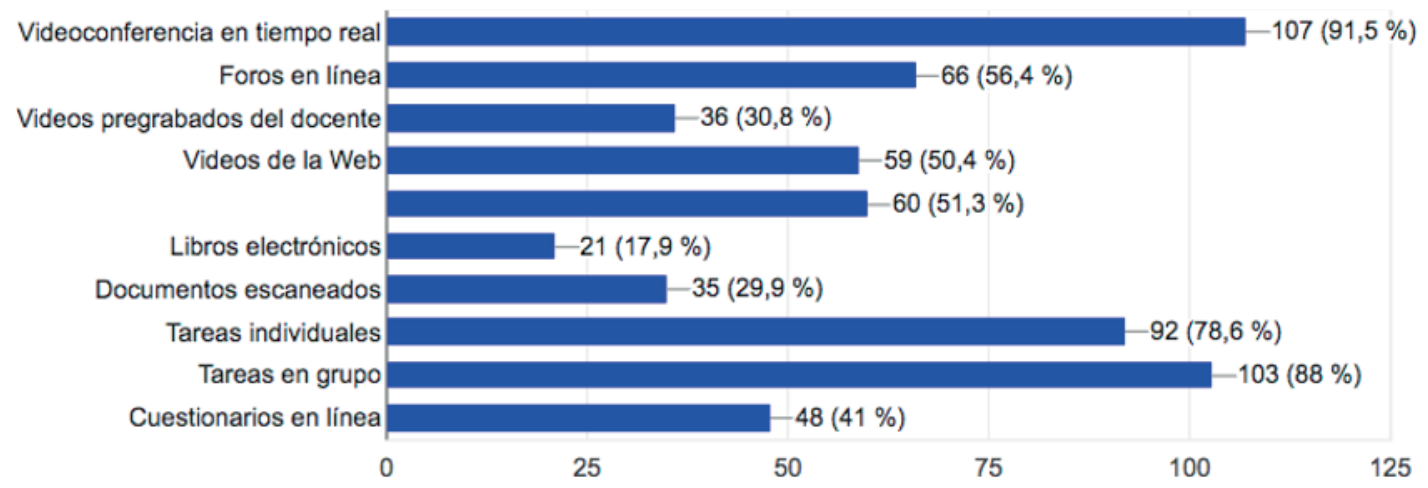

Figura 3. Estrategias de mediación pedagógicas sincrónicas y asincrónicas. Fuente: elaboración propia.

Según el criterio de la población estudiantil que completó el cuestionario, se reveló que el mayor inconveniente presentado frente al cambio de mediación pedagógica (de modalidad presencial a una remota con ayuda de las TIC) en tiempos de Covid-19 es la mala o nula conectividad con un $66,7 \%$. Además de que el $59 \%$ se queja de un exceso de tareas asincrónicas desproporcionales asignadas a la plataforma del campus virtual de la UTN. Esto ha provocado que el $42 \%$ presente dificultades para comunicarse. Además, el 47,9\% de los encuestados indican que al regresar a su casa aumentó el tiempo de colaboración en las tareas del hogar/empresas familiares. Una situación que sin duda alguna ha perjudicado significativamente el rendimiento académico por la persona estudiante.

A continuación, se evidencia en frases propias por las personas encuestadas, qué percepción les ha dejado el cambio de mediación pedagógica de modalidad presencial a una remota con ayuda de las TIC.

- Es una experiencia interesante, sería muy bueno que los profesores trataran de hacer las clases más dinámicas.

- No me gusta para nada.

- Muchas tareas y demasiada presión.

- Es realmente difícil, por la cantidad de tareas que dejan y las horas que hay que estar pegados en el computador recibiendo clases, y a veces la falta de conocimiento, de las plataformas tecnológicas aumenta la presión y el exceso de tareas.

- Normalmente 7 horas de estudio daría, sin contar las clases que hay que recibir, las tareas y proyectos pendientes. Y estar pendiente al computador por varias horas puede dañar la vista.

- Mala con respecto a trabajos que incluyen más recursos de lo que un estudiante podría costearse (switch, servidores, una computadora con más recursos), además de una mala conexión de Internet.

- En mi opinión ha sido algo cansado, ya que aparte de recibir bastantes horas en clase, también se dejan bastantes trabajos. Por lo que, algunas veces ha sido difícil por la cantidad de cosas por hacer.

- Es una modalidad interesante, pero no todos aprendemos al mismo ritmo, en clases uno se quedaba con el profesor para que le explicara mejor, pero en la virtualidad eso se complica. Ya sea porque el profesor tiene más clases o el estudiante tiene tareas en el hogar que debe cumplir.

- No me siento bien en esta modalidad virtual, sinceramente en otros cursos como en redes principalmente, casi siempre me siento frustrado por la cantidad de trabajos que deja este profesor, el tiempo de entrega de trabajos es limitado siento que el profesor de redes va volando en la enseñanza del curso, por lo cual uno va quedando perdido en el aprendizaje, el tiempo en que se asignan exámenes y pruebas cortas son para entregar en el mismo tiempo cosa que a veces no hay buena conexión y uno no cuenta con ese tiempo para la entrega inmediata. Con los demás cursos como en web, por ejemplo, excelente, se siente la comprensión de parte del profesor a los estudiantes excelente profesor la verdad lo felicito por la forma adecuada o su forma de enseñar siento que he apren-

Loáiciga, J. y Chanto, C. Valoración de las experiencias educativas en tiempos de COVID- 19 una oportunidad para innovar; caso estudiantes de la Universidad Técnica Nacional Sede Guanacaste. 
dido bastante en tan corto tiempo. Pero de manera en general por los motivos de mala conexión a Internet preferiría las clases presenciales, aunque estemos en tiempo de pandemia. El cambio es evidente más cuando no existe la comprensión por parte del profesor, aclaro no todos, pero en esta modalidad que estamos deberían de ser un poquito más comprensivos para la entrega de trabajos, prácticas y no hacernos sentir como si estuviéramos de manera presencial ya que no es así.

- No me gusta para nada esta modalidad, ya que no se cuenta con excelente conectividad a Internet, y muchas veces he perdido trabajos incluso exámenes por falta de conectividad y la $U$ no ayuda en nada a las personas que lo necesitamos.

- Han aumentado las tareas, y se torna abrumador y frustrante.

- No estamos listos para esta modalidad, ni la universidad, ni nosotros

- Sinceramente, no ha sido una experiencia tan buena. Aumentó el estrés por la cantidad de trabajos y el tiempo se limitó.

- En cuanto al área tecnológica, es una excelente opción ya que se aprovecha al máximo la enseñanza.

- Es más práctico, pero los profesores dejan muchos trabajos porque piensan que uno tiene más tiempo libre

- Es una metodología de mucho provecho, la facilidad de trabajar desde casa, ahorro de recursos (comida, pasajes, alquiler). Sin embargo, la parte de docencia recurrió a enviar trabajos muy extensos, en un promedio de 2 trabajos extensos más la participación en foros, lo cual perjudica al momento de matricular una malla curricular completa. Además, en ciertos cursos además de dejar extensos trabajos, utilizan 4 horas en clases por plataformas de teleconferencia.

- Otro aspecto es que no se tiene determinado una sincronización en las herramientas, existen profesores que utilizan Zoom, otros Teams o Meet. En ese caso sería bueno tener un esquema general en el uso de herramientas Office 365.

- Los cursos de sienten más pesados, cargados de tareas fuera de las clases virtuales

- Estar en comunidad con los compañeros y profesores motiva bastante a la hora de seguir estudiando. En modalidad virtual se vuelve menos "tentador"... y uno se siente más aislado.

- Es diferente porque siento que no hay mucha confianza en las videoconferencias, poca participación, las clases presenciales ayudaban a que todos nos ayudaremos a convivir entre sí y a compartir, además creo que las clases virtuales están generando en algunos aburrimiento y no debería ser así sino sacarle el máximo provecho porque aunque no sea de mi preferencia me estoy esforzando para cumplir mis objetivos.

- Mayor estrés y problemas al estar tanto tiempo sentado en la misma posición

- Pésima calidad de enseñanza de parte de la mayoría de los profesores, todo el aprendizaje recae sobre los estudiantes, ya que muchas veces tenemos que exponer nosotros mismos los contenidos del programa de estudio de la materia.

- Demasiada carga académica.

- Muchos trabajos por parte de los docentes, y poco tiempo para entregarlos.

- La modalidad presencial se puede jugar con diferentes factores, que aumenta la facilidad de aprendizaje.

- Ha influenciado de manera positiva y negativa, de manera positiva, ya que hemos aprendido a usar más aplicaciones tecnológicas, y a ser más creativos en presentaciones y trabajos, de manera negativa, ya que al ser una estudiante de Turismo deseo ir al campo a practicar lo teórico y no solo quedarme con eso con lo teórico, espero que para el próximo cuatrimestre ya vuelva todo presencial.

- Generalmente, el exceso de tareas o exposición es lo que torna más difícil con la modalidad virtual, aunque sea duro para nosotros los estudiantes no debemos rendirnos.

- Es bastante difícil adaptarse a los cambios, más cuando las diferentes situaciones que se salen de control como la conectividad de Internet que suele ser muy mala o saturarse, al igual que las Apps, trabajar y ayudar en actividades del hogar por el hecho de estar aquí, por esta razón se invade el espacio de estudio algo que no sucedía anteriormente que era presencial, sobrecargo de trabajos semanales y por ende la falta de compresión de algunos profesores al no poder unirse a una clase, mucho tiempo en la computadora también es agotador y los profesores no respetan el tiempo establecido, la verdad espero con ansias volver a la universidad presencial pronto.

- Lo único que sé, es que la Universidad Técnica Nacional no ayuda a los estudiantes ni en la modalidad presencial ahora menos en la virtual... Lo único que hacen es por el bien de ellos...

- No es lo mismo, desde mi punto de vista siempre será necesario la parte presencial, más cuando nos referimos a carreras donde la parte práctica es indispensable, ya que no es lo mismo salir solo y hacer sus propias observaciones, a poder estar con los compañeros y el docente compartiendo los conocimientos aprendidos en la parte teórica. 
A continuación, se muestra una síntesis de las variables encontradas durante el análisis de las percepciones indicadas por los encuestados.

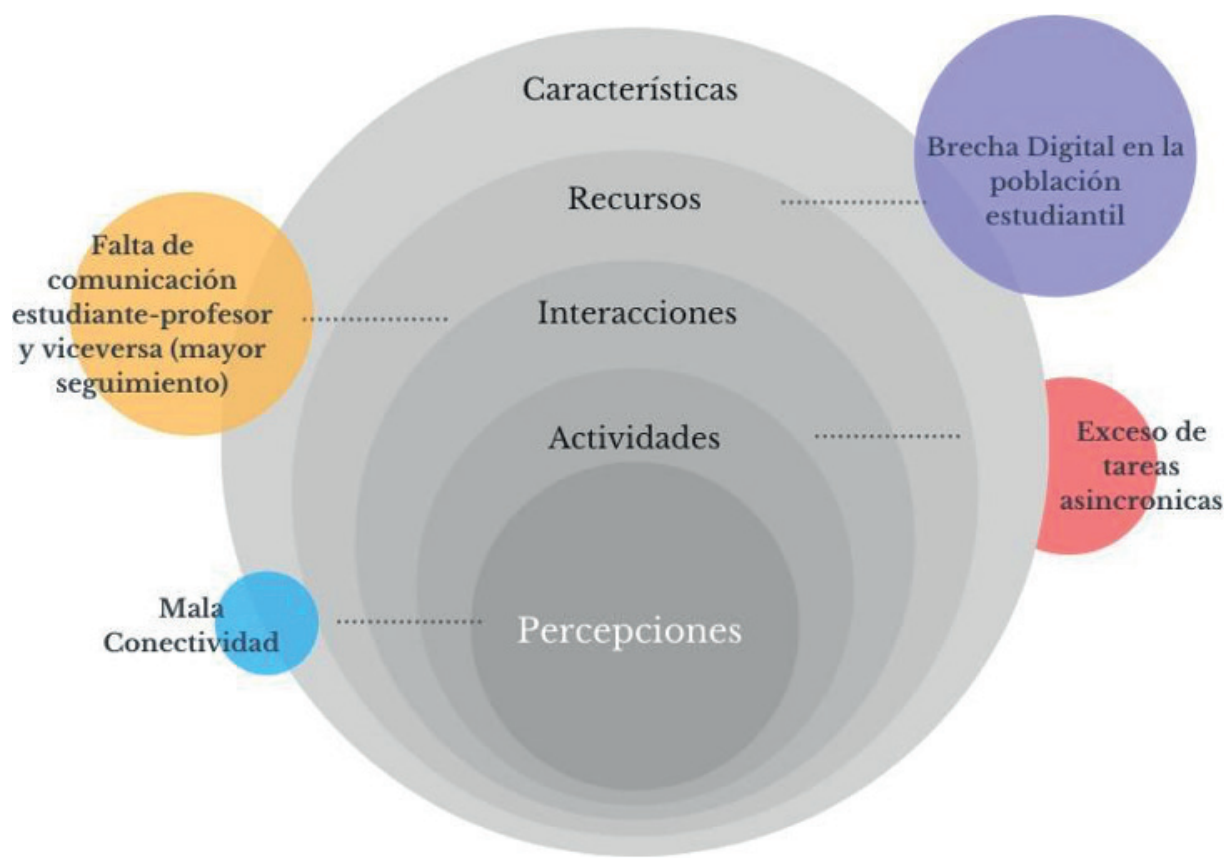

Figura 4. Síntesis de percepciones por el estudiantado encuestado. Fuente: elaboración propia.

\section{Conclusiones}

El mundo en general se enfrenta a una singular problemática sanitaria, que ha obligado a realizar acciones como el distanciamiento social y a la inmovilización de las distintas instituciones, con acciones como el teletrabajo. Pero, la educación no puede colocarse en un estado pasivo, esperando una posible vacuna para el Coronavirus SARS-CoV-2 y la enfermedad que causa la denominada Covid-19

Debido al contexto exhibido por la pandemia de la Covid-19, la UNESCO (2020) subraya que 990.925.124 estudiantes han sido afectados por esta situación. Por tal efecto, el docente debe adjudicarse un nuevo rol apoyado por las TIC, en donde tome en cuenta el aislamiento social preventivo necesario en las instituciones de educación.

Tal y como lo menciona Contreras-Hernández (2020, p. 9):

La manera de educar se está transformando, los sistemas presenciales están transitando a sistemas mixtos en donde convive la enseñanza presencial, las plataformas educativas y el uso de las TIC, además se ha ampliado la matrícula mediante sistemas de aprendizaje netamente virtuales. La transformación de la educación es irreversible, tanto en el uso de los medios como en los modelos educativos empleados, haciéndose necesario transitar de modelos clásicos a modelos constructivistas que se ajusten de mejor manera a la nueva realidad y permitan a los alumnos construir su propio saber.

Los resultados emanados plasmaron la evidencia de que el docente, debió adjudicarse una nueva función, un nuevo rol participado por las TIC frente al aislamiento obligatorio.

De igual forma se puede evidenciar el fortalecimiento de la utilización de las TIC en el contexto virtual del proceso de enseñanza-aprendizaje, generada por los docentes, estableciendo un nuevo lanzamiento al elemento formativo a partir de prácticas significativas e innovadoras.

Durante el estudio de la investigación, en los estudiantes de la Universidad Técnica Nacional, Sede Guanacaste se evidencia que la Covid-19 ha generado una abrupta transformación en el proceso de enseñanza-aprendizaje, por ende, las orientaciones deben tener como elementos de aprendizaje efectivo un currículo de educación con ayuda 
de herramientas tecnológicas, como valor agregado a su contenido curricular a partir del desarrollo de las competencias TIC en el crecimiento profesional docente.

Un efecto de la pandemia y lo que generó en la enseñanza en la educación a nivel universitario, es la actualización obligatoria de parte del personal docente y el estudiantado de nuevas herramientas tecnológicas para ser utilizadas en las clases.

El estudiantado manifiesta no estar $100 \%$ satisfecho en este nuevo paradigma remoto empezando porque se siente aislado de sus compañeros y el apoyo de la universidad, del todo, no ha sido el mejor, más de la mitad de los encuestados $78 \%$ considera que ha bajado su rendimiento académico (notas) por esta modalidad y en parte consideran que el personal docente necesita capacitarse más con herramientas tecnológicas.

Los resultados muestran, que existe una oportunidad de innovación, un nuevo paradigma en la educación superior pública de Costa Rica, la Universidad Técnica Nacional con Sede en Guanacaste debe de propiciar espacios que transmitan la necesidad de que el día después de la pandemia del Covid-19 este tipo de modalidades remotas, virtuales perduren hacia las nuevas generaciones.

Las TIC son aliadas y llegaron para facilitar la transmisión del conocimiento. Sin embargo, se debe de mejorar en puntos relacionados con la brecha digital y el ritmo de aprendizaje por el estudiantado y el desarrollo del contenido de los cursos adaptados en este tipo de modalidades.

\section{Referencias}

Amcham (10 de abril de 2019). ODS N4: Garantizar una educación inclusiva, equitativa y de calidad y promover oportunidades de aprendizaje durante toda la vida para todos. Amcham Costa Rica. https://www. amcham.cr/objetivo-n4-garantizar-una- educacion-inclusiva-equitativa-y-de-calidad-y-promover-oportunidades-de- aprendizaje-durante-toda-la-vida-para-todos/

Aguilar Ródenas, C. (2020). Educación, género y coronavirus. Revista con la A, 69, 1-5. http://repositori.uji. es/xmlui/bitstream/handle/10234/188634/70197. pdf? sequence $=1$ \&isAllowed $=\mathrm{y}$
Barrera Rea, V. F., y Guapi Mullo, A. (2018). La importancia del uso de las plataformas virtuales en la educación superior. Atlante Cuadernos de Educación y Desarrollo, julio. https://www.eumed. net/rev/atlante/2018/07/plataformas-virtualeseducacion.html

Contreras-Hernández, N. M. M. (2020). El reto del Covid-19 para la Educación en México. Revista Buen Gobierno, 6. https://doi.org/10.35247/buengob 2906

Delgado, J., García, C., Guaicha, K., y Prado, M. (2020). La Webquest como herramienta didáctica para potenciar el pensamiento crítico en la formación de estudiantes universitarios. Revista Tecnológica-Educativa Docentes 2.0, 9(1), 49-55. https:// doi.org/10.37843/rted.v9i1.96

García-Pérez, Ángela, y Mendía, Rafael (2015). Acompañamiento Educativo: El rol del educador en Aprendizaje y Servicio Solidario. Profesorado. Revista de Currículum y Formación de Profesorado, 19(1),42-58. [fecha de Consulta 3 de Noviembre de 2020]. ISSN: 1138-414X. https://www.redalyc.org/articulo.oa?id=567/56738729004

GEM, I. (2020, marzo 26). ¿Cómo están abordando los países los desafíos del Covid-19 en materia de educación? Una instantánea de las políticas aplicadas. Blog de la Educación Mundial.

https://educacionmundialblog.wordpress. com/2020/03/26/como-estan-abordando- los-paises-los-desafios-del-covid-19-en-materia-de-educacion-una-instantanea-de- las-politicas-aplicadas/

Guaman-Chávez, R. (2020). El Docente en Tiempo de Cuarentena. Revista Tecnológica- Educativa Docentes 2.0, 8(2), 21-27, DOI: https://doi. org/10.37843/rted.v8i2.154

Hueso, L. C. (2020). La enseñanza digital en serio y el derecho a la educación en tiempos del coronavirus. Revista de Educación y Derecho., 0(21), Article 21. https://revistes.ub.edu/index.php/RED/article/ view/31213

Iglesia-Villasol, M. C. (2019). Huellas de los estudiantes en las plataformas virtuales. Aplicación para evaluar una metodología de aprendizaje activo.: Footprints of students on virtual platforms. Application to evaluate an active learning methodology. Revista Electrónica Interuniversitaria de For- 
mación del Profesorado, 22, 173-191. https://doi. org/10.6018/reifop.371341

Sandoval, C. H. (2020). La Educación en Tiempo del Covid-19 Herramientas TIC: El Nuevo Rol Docente en el Fortalecimiento del Proceso Enseñanza Aprendizaje de las Prácticas Educativa Innovadoras. Revista Tecnológica-Educativa Docentes 2.0, 9(2), 24-31. https://doi.org/10.37843/rted. v9i2.138

UNESCO. (2020). Apoyar a los docentes en los esfuerzos para facilitar la vuelta a la escuela Orientaciones para los responsables de la formulación de políticas. Educación 2030. https://unesdoc.unesco.org/ ark:/48223/pf0000373479_spa

UNESCO. (16 de marzo de 2020). ¿Cómo estás aprendiendo durante la pandemia de COVID-19? UNESCO. https://es.unesco.org/covid19/educationresponse

Zeballos, M. (2020). Acompañamiento Pedagógico Digital para Docentes. Revista Tecnológica-Educativa Docentes 2.0, 9(2), 192-203.https://doi. org/10.37843/rted.v9i2.164 